\title{
Sintomatología de depresión, ansiedad y baja autoestima en mujeres obesas con trastorno del comedor compulsivo
}

\author{
Symptoms of depression, anxiety and \\ low self-esteem in women with obesity \\ and binge eating disorder
}

\begin{abstract}
TThe objective of this study was to determine the presence of symptoms of depression, anxiety and low self-esteem in women with obesity and binge eating disorder. 60 women using the primary health care system in Lautaro were selected. Within the inclusion criteria were identified diagnosis of nutritional obesity and the presence of binge eating disorder without medical and mental disorders. Depression Scale Anxiety of Goldberg and Rosenberg Self-Esteem Scale were applied individually. For descriptive analysis results were used, through a frequency distribution and $\mathrm{chi}^{2}$ test. Among the findings it was noted that this group had mostly symptoms of depression and anxiety, which correlates with the literature. However, low self-esteem, which contrasts with the evidence examined, was not observed. This study shows that obesity is not just a simple symptom, but there are psychopathological backgrounds like depression and anxiety to consider, which should be incorporated into the comprehensive diagnosis in its primary health care plan for later multidisciplinary intervention.
\end{abstract}

Key words: Depression, anxiety, low self-esteem, obesity, binge eating disorders.

\section{INTRODUCCIÓN}

Actualmente la obesidad constituye un serio problema de salud pública tanto en Chile, como en varios otros países; entendiéndose por obesidad, un índice de masa corporal mayor o igual a $30 \mathrm{~kg} / \mathrm{m}^{2}$. Una de sus principales causas es una ingesta excesiva de alimentos calóricos y/o disminución de actividad física. La obesidad es el factor principal de riesgo de enfermedades crónicas no transmisibles en nuestra sociedad (1). Según la Encuesta Nacional de Salud 2009-2010, existe $25,1 \%$ de la población con este diagnóstico, alcanzando 30,7\% en las mujeres y $19,2 \%$ en los hombres (2). En la salud pública actual existen protocolos de atención para contrarrestar el exceso de peso: atención nutricional, promoción de salud, entre otros, sin embargo no se considera la alternativa de que existan trastornos alimentarios que pueden estar en comorbilidad con este estado nutricional, porque cuando se habla de ello se asocia rápidamente a lo más conocidos (anorexia y bulimia).

Los trastornos de conducta alimentaria son problemas psiquiátricos, que se definen como una alteración persistente del comer o de conductas relacionadas con esta acción, que se
Fabiola Andrea Godoy L.

Unidad de Nutrición, CESFAM Cristóbal Sáenz Cerda Universidad de La Frontera, Temuco, Chile

Dirigir la correspondencia a: Nutr. Fabiola Godoy Leal CESFAM Cristóbal Sáenz Cerda, Lautaro, Chile Télefono: (56-45) 2996581

E-mail: fabiolagodoyleal@gmail.com

Este trabajo fue recibido el 15 de Mayo de 2014 y aceptado para ser publicado el 11 de Agosto de 2014.

traducen en una alteración en el consumo o absorción de los alimentos, que alteran en forma significativa la salud física o el funcionamiento psicosocial, y no son secundarios a ningún trastorno médico general u otro síndrome psiquiátrico (3).

El Diagnostic and Statistical Manual of Mental Disorder cuarta edición (DSM IV-tr), clasifica los Trastornos de Conducta Alimentaria en Anorexia, Bulimia y Desórdenes Alimentarios No Especificados (4). En esta última categoría existen desórdenes o trastornos menos conocidos, uno de éstos es el Trastornos Comedor Compulsivo (TCC), (Binge Eating Disorder), el cual se define como: ingesta de grandes cantidades de comida asociado con indicadores subjetivos y comportamiento de pérdida de control; angustia significativa por la alimentación compulsiva; y la ausencia del uso regular de comportamientos compensatorios inapropiados: purgaciones, ayunos y ejercicios excesivos (5). El TCC no es un trastorno muy conocido en nuestro país, sin embargo en otros países se han realizado estudios en población de mujeres con obesidad que concluyen la existencia de una prevalencia en pruebas clínicas; en Europa de 10-15\% y Estados Unidos entre 20-30\% (6). En 
esta investigación consideraremos como variable poseer TCC, con la finalidad de demostrar que existen mujeres que pueden ser pesquisadas con este trastorno desconocido.

Existen antecedentes de la presencia de factores psicopatológicos en anorexia y bulimia, dentro de ellos sintomatología depresiva y ansiedad, en la que existe una correlación positiva con dichos trastornos (7). Estos mismos factores se encuentran en estudios en personas con sobrepeso y obesidad $(8,9)$. Por ello nos interesó conocer si existen estos mismos factores en personas con obesidad y además que posean el trastorno del comedor compulsivo.

Debemos tener en cuenta que la depresión, es una alteración patológica del estado de ánimo, caracterizada por un descenso del humor. La prevalencia nacional de síntomas depresivo es $17,2 \%$, siendo mayor en mujeres $(25,7 \%)$ que en hombres (8,5\%) (2). El segundo factor es la ansiedad la que puede ser comprendida como la contraparte patológica del miedo normal, y se manifiesta como alteraciones del ánimo, de los pensamientos, del comportamiento y de actividades fisiológicas (4).

Otra variable a abordar es la autoestima que es considerada como un factor relevante en el pronóstico de los trastornos de conducta alimentaria, puesto que refleja un pensamiento negativo hacia el individuo mismo, así como baja autoestima y fluctuaciones en el estado de ánimo (10). Se establece que la existencia de una autoestima normal proporciona un seguro a la capacidad de alcanzar un mejor afrontamiento de los conflictos y reducción de peso en personas con obesidad (11). En nuestro país se realizó un estudio para observar su presencia en personas con obesidad, en la cual el grupo fue asociado positivamente con baja autoestima (12). Nuestro estudio lo realizaremos con mujeres, por su alta prevalencia en algunos factores de estudio.

El objetivo de este estudio fue determinar la presencia de sintomatología de depresión, ansiedad y autoestima en mujeres con obesidad y que posean trastorno del comedor compulsivo.

\section{SUJETOS Y MÉTODOS}

El diseño de la investigación es exploratorio descriptivo de corte transversal. La población que consideramos estuvo conformada por las beneficiarias del Programa de la Mujer del CESFAM Cristóbal Sáenz Cerda de Lautaro. La muestra se seleccionó por conveniencia hasta alcanzar un tamaño muestral de 60 mujeres obesas (20 por variable). Los criterios de inclusión fueron los siguientes: mujeres con obesidad $\left(I M C \geq 30 \mathrm{~kg} / \mathrm{m}^{2}\right)$, entre 20 a 65 años, sin patologías médicas y mentales diagnosticadas y con trastorno del comedor compulsivo. Para ello consideramos a las beneficiarias de salud que asistieron a consulta nutricional, por primera vez, entre los meses de diciembre de 2009 y enero 2010. A cada mujer con diagnóstico de obesidad, antes de realizar intervención nutricional, las invitamos a participar de este estudio a través de un consentimiento informado, previamente validado por un comité de Etica. A las personas que aceptaron les aplicamos los criterios del DSM-IV tr para identificar a una persona con el trastorno del comedor compulsivo, la cual es una escala en la que debe presentar al menos 3 de los siguientes hallazgos asociados: (a) comer más rápido de lo normal; (b) comer hasta sentirse muy saciado; (c) ingesta de grandes cantidades sin hambre; (d) comer sólo por vergüenza (e) disgusto, depresión o culpa por los patrones alimentarios (4). Un total de 81 mujeres realizaron este instrumento, pero 60 fueron las que clasificaron dentro de este trastorno y consideramos para este estudio.
Les registramos los datos personales, como: edad, peso, talla, IMC y posteriormente les aplicamos la escala de Depresión y Ansiedad del Cuestionario de Goldberg que consiste en dos subescalas de 9 preguntas, todas ellas con respuestas dicotómicas (si/no). Su aplicación es intercalada en el contexto de la entrevista clínica, en la que se interroga al paciente sobre si ha presentado en las últimas dos semanas algunos de los síntomas a los que hace referencia las preguntas. La escala se estructura en 4 preguntas de despistaje, para determinar si es o no probable que exista un trastorno mental, y un segundo grupo de 5 ítems que se formulan sólo si se obtienen dos o más respuestas positivas. El corte de diagnóstico positivo es de $\geq 4$ puntos. Este instrumento está diseñado para detectar "probables casos" de pacientes portadores de un trastorno depresivo y/o ansiedad, no para diagnosticarlos. La escala global tiene una sensibilidad del $83 \%$ y una especificidad del $82 \%$. (13)

Para evaluar autoestima se utilizó la Escala de Rosenberg, validación española, consistentes en 10 preguntas con respuestas tipo likert con indicadores de muy en desacuerdo a muy de acuerdo, con puntaje de 1 a 4 puntos, con 5 reactivos inversos $(1,3,4,6$ y 7). El puntaje final se suma clasificándose en 30 a 40 puntos autoestima elevada, de 26 a 29 puntos autoestima media y $<25$ puntos autoestima baja. La consistencia interna de la escalas se encuentran entre 0,76 y 0,87. La fiabilidad es de 0,80 . (14)

Se ingresaron los datos a una planilla del software estadístico SPSS 13.0, a través del cual se realizaron todos los procedimientos estadísticos. Se realizó un análisis descriptivo, a través de una distribución de frecuencias, para obtener el porcentaje de mujeres con síntomas de depresión, ansiedad y autoestima de nivel baja, media o alta. Se realizó una prueba de chi $^{2}$, para evaluar si los resultados eran o no significativos.

A todas las usuarias incluidas en el estudio, como aqueIlas que no fueron consideradas para tal efecto, les aplicamos todos los instrumentos detallados y les entregamos atención integral con psicólogo, médico y educación con nutricionista.

\section{RESULTADOS}

La muestra de estudio estuvo constituida por 60 mujeres. La edad fluctuó entre los 20 y 63 años con una media de 39 años. El índice de masa corporal se encuentra entre los 30,0 y $44,9 \mathrm{~kg} / \mathrm{m}^{2}$, con una media de $34,7 \mathrm{~kg} / \mathrm{m}^{2}$.

El $65 \%$ de las mujeres presentaron sintomatología depresiva y $53.3 \%$ presentaron ansiedad. Un 23,3\% presentaron autoestima baja, $21.7 \%$ autoestima media y $55 \%$ autoestima alta (normal). En la tabla 1 se incluye la prueba Chi cuadrado de las variables sintomatología depresiva, ansiedad y autoestima del grupo de estudio.

\section{DISCUSIÓN}

En los resultados se observó que existió la presencia de sintomatología depresiva y ansiedad, lo que se correlaciona con otros estudios que establecen la presencia de esta sintomatología en pacientes obesos y con trastorno del comedor compulsivo (15). En cuanto a la depresión existen antecedentes que las personas con esta patología tienden a disminuir el apetito (9), sin embargo hay personas que les sucede lo contrario, lo que se explica, por que en el trastorno depresivo se puede alterar seriamente la relación con la comida. Las ideas de fracaso, inferioridad o culpa por no alcanzar un ideal son capaces de producir depresión. Uno de los motivos que una persona puede utilizar para hacerse autorreproches continuos es intentar adelgazar y no conseguirlo. Hay personas que 
desean adelgazar, pero enseguida sienten ganas de comer. Después de hacerlo sienten que no tienen fuerza de voluntad y que no podrán llegar a ser como quieren porque no tienen el cuerpo que desean, entonces se deprimen (16).

En cuanto a la ansiedad, existen antecedentes que se asocian a personas con exceso de peso. Cuando la ansiedad sobrepasa determinados límites y empieza a manifestarse una tendencia repetitiva de comer a destiempo, para calmar un estado de malestar, aumentando el aporte calórico que necesitamos (16). Si analizamos el porcentaje de obesidad que tenemos en la actualidad en nuestro país, nos empezamos a preguntar ¿cuántas de esas personas tienen ansiedad?, en nuestro estudio observamos que más de la mitad del grupo en estudio presenta esta sintomatología, pero lo que no podemos concluir si esta fue la causante de la obesidad, sería interesante abordar en futuros estudios las causas de la ansiedad en la alimentación.

El segundo hallazgo importante, es que no se observó que la baja autoestima sea predominante en el grupo que posee depresión y ansiedad, por el contrario la mayoría de las mujeres del estudio poseen autoestima normal. La autoestima se propone que sea considerada como un factor relevante en el pronóstico de los trastornos de conductas alimentarias (11). Por ello es importante aplicar un instrumento de evaluación de autoestima, ya que si posee un nivel normal de esta condición se podría trabajar fomentando más la confianza en sí mismo y la motivación para llevar a cabo los cambios de conductas alimentarias. Una autoestima normal es una característica de quienes tienen una buena aceptación de sí mismos y un buen equilibrio emocional, que son capaces de disfrutar situaciones sociales, pero también de la soledad, que tienen autoconfianza, menor temor ante los fracasos, pocos pensamientos negativos y buena motivación (17). Si posee autoestima media o baja, se debe abordar de forma intensiva e integral para mejorarla como factor protector.

Al aplicar la prueba de $\mathrm{chi}^{2}$, se observó que los resultados no eran significativos, por lo que se evaluó que el número de participantes en el estudio era insuficiente. Se considerará aumentar el número de participantes en la investigación para un posterior estudio.

La importancia de este estudio es observar la presencia de varios factores en la obesidad, uno de ellos es el trastorno de comedor compulsivo, que en muchos países se identifica a la población en riesgo, a la cuales se ha identificado los factores que causan este trastorno (18 - 19) y les han realizado estrategias de intervención efectivas (20), el cual sería interesante abordar en Chile. Otro punto es el hallazgo de factores psicopatológicos de depresión y ansiedad en población con obesidad, que actualmente en la salud publica es muy poco abordado en personas con malnutrición por exceso. Como también considerar conocer el nivel de autoestima de las personas, ya sea para ser un factor protector o para reforzarla en el tratamiento integral.

Debemos reflexionar que la etiología de la obesidad es diversa, sin embargo proponemos abordarlo como un síntoma multifactorial, en el cual se debe indagar todas las causas posibles, entre ellas evaluar si existe un trastorno alimentario asociado y si existen factores psicopatológicos (depresión, ansiedad y autoestima) que nos permitan planificar estrategias de intervención multidisciplinaria en la atención primaria de salud.

\section{RESUMEN}

El objetivo de este estudio fue determinar la presencia de sintomatología de depresión, ansiedad y baja autoestima en mujeres con obesidad y trastorno del comedor compulsivo. Se seleccionaron a 60 mujeres usuarias del sistema de atención primaria de salud de la comuna de Lautaro. Dentro de los criterios de inclusión se determinaron: diagnóstico nutricional de obesidad, sin patologías médicas y mentales diagnosticadas y la presencia del Trastorno del Comedor Compulsivo. Se les aplicaron aplicaron de manera individual la Escala de Depresión y de Ansiedad de Goldberg y la Escala de Autoestima de Rosenberg. Para los resultados se utilizó un análisis

TABLA 1

Distribución de frecuencia de sintomatología depresiva, ansiedad y autoestima.

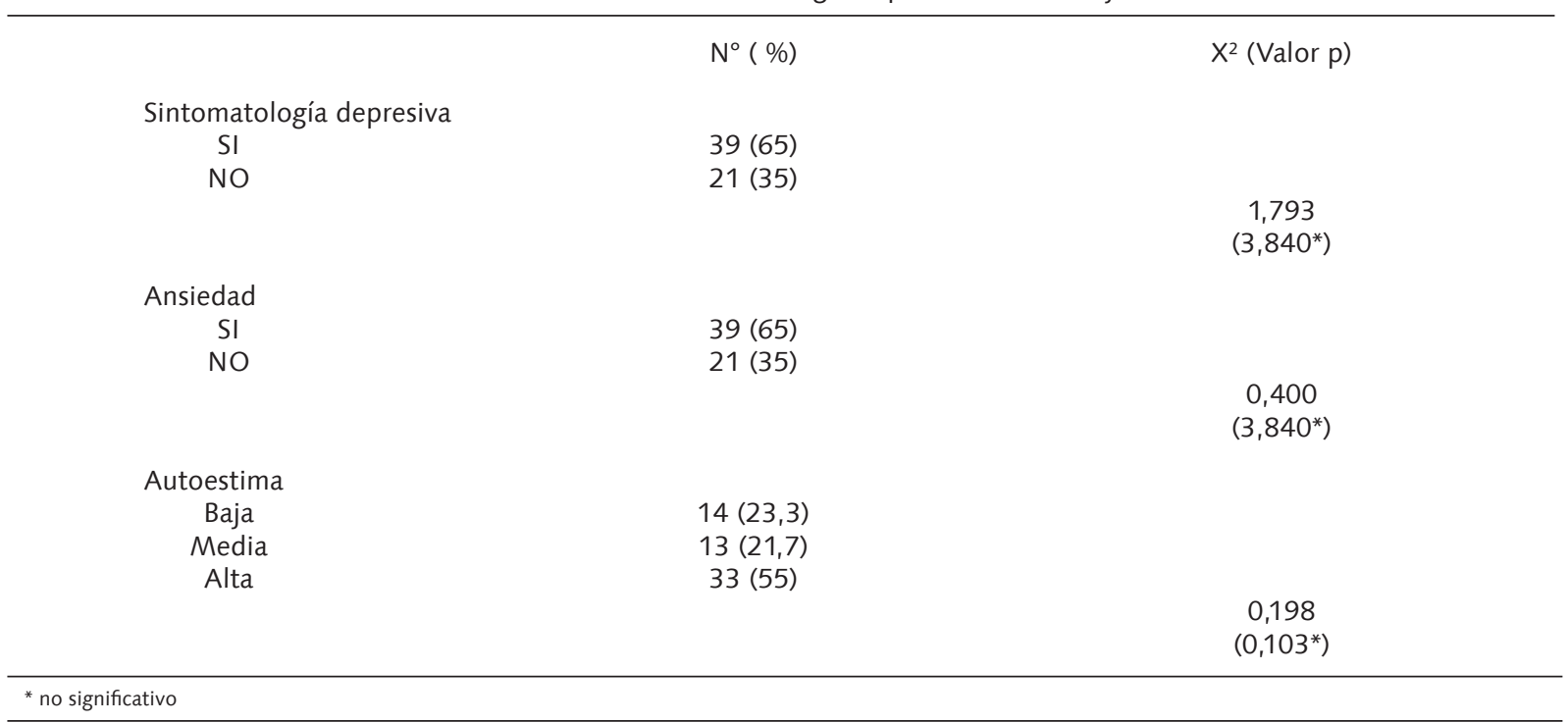


descriptivo, a través de una distribución de frecuencias y una prueba de $\mathrm{chi}^{2}$. Se observó que este grupo mayoritariamente si presentaba sintomatología de depresión y ansiedad, que se correlaciona con la literatura. Sin embargo, no se observó una baja autoestima, que se contrapone con los antecedentes analizados. Este estudio, nos muestra que la obesidad no es tan sólo un síntoma aislado, sino que existen antecedentes psicopatológicos a considerar: como depresión y ansiedad, el cual se debería incorporar dentro del diagnóstico integral en el equipo de atención primaria de salud para un posterior plan de intervención multidisciplinario.

Palabras clave: depresión, ansiedad, baja autoestima, comedor compulsivo.

\section{BIBLIOGRAFÍA}

1. World Health Organization 2006. Organización Mundial del la Salud. Disponible en http://www.who.int/mediacentre/factsheets/fs311/es/index.html

2. Ministerio de Salud (2010). Encuesta Nacional de Salud 2009-2010. Extraído el 08 de enero de 2011 desde http://web.minsal.cl/portal/url/item/bcb03d7bc28b64dfe040010165012d23.pdf

3. Walsh, T. Atypical eating disorder in: Eating Disorder and Obesity. The Guilford Press 1995; 24: 135-40.

4. American Psychiatric Association. Diagnostic and Statistical Manual of Mental Disorder. Washington D.C., Estados Unidos: American Psychiatric Association. p 185. 1994.

5. Masheb, R., y Grilo, C. Binge eating disorder: A need for additional diagnostic criteria. Comprehensive Psych. 2000; 41: 159-162.

6. Ramacciotti, C., Coli, E., Passaglia, C., Lacorte, M., Pea, E., y Dell'Osso, L. Binge eating disorder and psycopathological features in a clinical sample of obese people in Italy. Psych Res. 2000; 94: 131-8.

7. Behar, R., Barahona, M., Casanova, D. e Iglesia, B. Trastorno depresivo, ansiedad social y su prevalencia en trastorno de conducta alimentaria, Rev Chil Neuro-Psiq. 2007; 45: 211-20.

8. Tapia, A. Ansiedad, un importante factor a considerar para el adecuado diagnóstico y tratamiento de pacientes con sobrepeso y obesidad. Rev Chil Nutr. 2006; 33: 352-7.
9. Masson, L. y Tapia, A. Detección de síntomas depresivos en pacientes con sobrepeso y obesidad. Rev Chil Nutr. 2006; 33: 162-9.

10. Goldschmidt, A., Hilbert, A., Manwaring, J., Wilfley, D., Pike, K., Fairburn, C., et al. The Significance of Overvaluation of Shape and Weight in Binge Eating Disorder. J Behav Res Therapy 2009; 48: 187-93.

11. Beato, L. y Rodríguez, T. (2004). Relación de las fases del cambio con la autoestima en pacientes con trastornos de la conducta alimentaria: seguimiento a un año. Psiquiatría. com. Extraído el 02 de junio de 2010 desde http://www. psiquiatría.com/artículos/tralimentación/14883

12. Alvarez, R. Obesidad y autoestima. Mc Graw Hill Interamericana. Ciudad de México, México, 1998.

13. Goldberg D, Bridges $K$, Duncan-Jones $P$, et al. Detecting anxiety and depression in general medical settings. Br Med J. 1988; 97: 897-9.

14. Rosenberg $M$, Schooler $C$, Schoenbach $C$, Rosenberg $F$. Global self-esteem and specific self-esteem. Amer Socio Rev 1995; 60: 141-56.

15. Dahl, J., Eriksen, L., Vedul-kjelsas, E., Strommen, M., Kulseng, B., Marvik, R. and Holen, A. Depression, anxiety and neuroticism in obese patients waiting for bariatric surgery: Differences between with and without eating disorder and subthreshold binge eating disorder. Obes Res Clin Practice. 2012; 6: 139-47.

16. Menéndez, I. Alimentación emocional. En Random House. Barcelona, España, 2006.

17. Alvarado, A., Guzmán, E. y González, M. (2005) Obesidad: ¿Baja autoestima? Intervención psicológica en pacientes con obesidad. Enseñanza e investigación en Psicología. 2005; 10: 417-28.

18. Fairburn, C., Doll, H., Welch, S., Hay, P., Davies, B., and O'Connor, M. Risk Factors for Binge Eating Disorder. Am Med Assoc. 1998: 55; 425-32.

19. Hilbert, A., Pike, K., Goldschmidt, A., Wilfley, D., Fairburn, C., Dohm, F., Walsh, T. and Streigel, R. Risk factors across de eating disorders. Psych Res. 2014: 54; 165-78.

20. Wilson, T., Wilfley, D., Agras, S. and Bryson, S. Psychological Treatments of Binge Eating Disorder. Arch Gen Psych. 2010: 67; 94-101. 